\title{
College Students' Knowledge of Risk Factors Related to Cardiovascular Disease
}

\author{
Christine Andrew ${ }^{1}$, Deborah Hiles-Gaddy ${ }^{2}$, Mikayla Lauren MacRitchie ${ }^{3} \&$ Sam Abraham $^{3}$ \\ ${ }^{1}$ Oncology Nursing, Intermountain Medical Center, Murray, UT, USA \\ ${ }^{2}$ Public Health Nurse, Saint Joseph County Health Department, Mishawaka, IN, USA \\ ${ }^{3}$ School of Nursing, Bethel College, Mishawaka, IN, USA \\ Correspondence: Sam Abraham, 9910 US Highway 31, Berrien Springs, MI 49103, USA.
}

Received: September 19, 2017

Accepted: October 1, 2017

Online Published: October 16, 2017

doi:10.20849/ijsn.v2i2.196

URL: https://doi.org/10.20849/ijsn.v2i2.196

\begin{abstract}
Background: Cardiovascular disease (CVD) is a prevalent health issue, accounting for a large proportion of deaths worldwide. Despite the high prevalence of this potentially detrimental condition, many college students are not fully aware of its contributing risk factors. The purpose of the current study was to determine college students' knowledge of risk factors related to CVD.

Methods: This was a quantitative, cross-sectional study with a descriptive design. A survey with 5 demographic questions and 20 Likert-type statements was administered to 118 students in a Midwestern college in the United States.

Results: The majority of the students displayed knowledge of risk factors such as smoking tobacco, inactive lifestyle, hypertension, low intake of fruits and vegetables, elevated cholesterol levels, high dietary sodium and obesity. However, a knowledge deficit existed regarding gender and ethnicity, which are non-modifiable risk factors, albeit important considerations.

Conclusions: Although students appeared knowledgeable about areas such as cholesterol in cardiovascular health, further education could be beneficial to improve this knowledge base.

Keywords: risk factors, cardiovascular disease, modifiable risk factors, non-modifiable risk factors, dietary risk factors, and heart disease

\section{Introduction}

Despite the high prevalence of cardiovascular disease (CVD), knowledge of its risk factors and corresponding preventative interventions may be inadequate in a given population. Possessing knowledge about the non-modifiable and modifiable risk factors of CVD is essential for the prevention of its development. Prevention may begin with alterations to modifiable factors to reduce the risk of development, especially if one has a significant risk because of non-modifiable factors. Understanding this at any age is important for optimal cardiovascular health. The purpose of this study was to determine college students' knowledge of risk factors related to CVD. A thorough analysis of literature regarding previous research on CVD risk factors is also included.
\end{abstract}

\section{Background}

Cardiovascular disease is a rapidly growing problem in the United States, serving as the "leading global cause of death, accounting for 17.3 million deaths per year" (Mozaffarian et al., 2015, p. 1). This statistic is expected to increase to more than 23.6 million by 2030 (p. 1). The current prevalence of CVD and its projected increase indicates the true severity of this issue. The majority of the problem could be related to knowledge deficits and lack of motivation to make changes in modifiable risk factors.

Mozaffarian et al. (2015) conducted a study about the prevalence and incidence of heart disease and discovered that "Heart disease is the No. 1 cause of death in the world and the leading cause of death in the United States, killing over 375,000 Americans a year" (p. 1). One out of every seven deaths in the United States currently can be attributed to CVD, with more than 2,150 Americans dying of CVD every day. This accounts for an average of 1 death every 40 seconds (p. 1$)$. 
Many behaviors that increase the risk for CVD are modifiable, such as obesity, smoking, unhealthy diet, physical inactivity, hypertension, and high cholesterol. Despite the potential for modifications, the prevalence and control of traditional risk factors remain an issue for many Americans. Increased education about these risk factors may contribute to the reduction of CVD in the United States each year. Understanding one's knowledge of CVD and its corresponding risk factors is an important step towards its reduction in the population.

\section{Review of the Literature}

The literature collected for this study was obtained using the college library EBSCO host database, specifically the Cumulative Index to National and Allied Health Literature. The articles selected contain evidence-based practice and were published between 2010 and 2015. Keywords used in the search were risk factors, cardiovascular disease, modifiable risk factors, non-modifiable risk factors, dietary risk factors, and heart disease.

\subsection{Elevated Cholesterol Levels}

Patel, Winkel, Ali, Narayan, and Mehta (2015) defined elevated cholesterol as "a measured total blood cholesterol level of at least $6.21 \mathrm{mmol} / \mathrm{L}(\geq 240 \mathrm{mg} / \mathrm{dL}$ ) or use of medication" (p. 246). Ignatavicius and Workman (2013) noted that consuming a diet high in fat and triglycerides leads to elevated cholesterol levels. Excessive levels of cholesterol in the blood can lead to its accumulation on the walls of arteries. This condition, known as atherosclerosis, causes a narrowing of the blood vessels, reducing blood flow to the heart. When the heart does not receive adequate perfusion, ischemia and infarction can occur. Atherosclerosis is a leading risk factor for cardiovascular disease (pp. 772-773).

Melnyk, Panza, Zaleski, and Taylor (2015) conducted a study among college freshmen to assess awareness and knowledge of cardiovascular risk through blood pressure (BP) and cholesterol testing. The participants completed three study visits. At the first visit, they were given questionnaires assessing their knowledge of risk factors contributing to CVD; they also had their BP checked and provided a blood sample for a complete lipid profile (total cholesterol, high-density lipoprotein, low-density lipoprotein, and triglycerides). During the second visit, one month after the first, they received approximately 15 minutes of individual education regarding their BP and lipid profile results and additional informational brochures. About a month later they returned for a third session to repeat the questionnaires. Results of the study determined that the educational intervention improved the students' knowledge of CVD (pp. 139-141).

\subsection{Diabetes}

Diabetes mellitus (DM) is chronic high blood glucose levels in the blood, known as hyperglycemia. Problems with insulin secretion, action, or both, lead to the elevated levels of blood glucose (Ignatavicius \& Workman, 2013, p. 1411). DM was defined by Patel et al. (2015) as a measured HbAlc level of at least $6.5 \%$ or use of medication (p. 246). Ignatavicius and Workman (2013) claimed severe diabetes could cause premature and severe atherosclerosis from microvascular damage. This may occur from the arterial damage caused by hyperglycemia. Additionally, diabetes causes an increase in low-density lipoprotein cholesterol (LDL-C) and triglyceride levels in the blood. Those diagnosed with DM are at an increased risk for CVD and may have a reduced lifespan consequently (pp. $773 \& 1413$ ).

\subsection{Hypertension}

Hypertension (HTN), or elevated blood pressure, is an average systolic blood pressure equal or greater than 140 $\mathrm{mm} \mathrm{Hg}$, an average diastolic blood pressure greater or equal than $90 \mathrm{~mm} \mathrm{Hg}$, or dependent use of antihypertensive medication (Patel et al., 2015, p. 246). Ignatavicius and Workman (2013) explained how HTN increases the workload of the heart, thereby increasing the risk for myocardial infarction (MI) and heart disease. If one has DM or heart disease, his/her blood pressure should be kept below 130/90 (p. 775).

Cardiovascular mortality is associated with five leading modifiable risk factors. Patel et al. (2015) compared the contribution of elevated cholesterol levels, DM, HTN, obesity, and smoking to cardiovascular mortality for each state in the nation. They determined HTN and high cholesterol were the most common risk factors among both men and women. Patel et al. found that about half of national cardiovascular deaths could be connected to the combination of those five risk factors. The states that had a high prevalence of one risk factor also appeared to exhibit a high prevalence of others as well. It was also observed that states performing poorly demonstrated multiple risk factors simultaneously. By removing all of the risk factors collectively, Patel et al. (2015) believed that they could prevent up to $54 \%$ of cardiovascular deaths in the nation (pp. 248-249). 


\subsection{Obesity and Sedentary Lifestyle}

Obesity is determined by using height and weight to compute body mass index (BMI). A person with a BMI of at least $30 \mathrm{~kg} / \mathrm{m}^{2}$ is considered to be obese (Patel et al., 2015, p. 246). To support the claim that a sedentary lifestyle increases your risk for CVD, Kozey-Keadle et al. (2014) conducted a study to analyze the impact of exercise on the cardiovascular system. They assessed the benefits of moderate to vigorous exercise, as compared to a more sedentary lifestyle, where the participant's exercise consisted of activities like moving around the house or place of work (p. 771). One group was encouraged to merely stand up during commercial breaks while watching television, or stand up every hour while at work. This group was instructed not to exercise and only perform small increments of movement to decrease sedentary time. Conversely, the second group was advised to implement frequent exercise. This group was encouraged to exercise on a treadmill or stationary bicycle for 40 minutes a day, five times a week while monitoring heart rate. The third group was instructed to incorporate both interventions by reducing sedentary time and exercising occasionally. Dietary factors remained the same for both groups to control extraneous variables (Kozey-Keadle et al., 2014, pp. 771-772).

As a result of this study, Kozey-Keadle et al. (2014) found that reducing sedentary time and incorporating moderate to vigorous exercise decreased BMI levels dramatically. A decrease in systolic blood pressure was noted in all groups; a diastolic decrease was noted in the group that decreased sedentary time without exercise, and a decrease in triglycerides was noted in the group that was encouraged to only incorporate exercise (p.774). Kozey -Keadle et al. determined that to significantly decrease the risk of CVD; it is imperative to maintain an active lifestyle by decreasing the amount of sedentary time while incorporating a moderate level of exercise five days a week for at least 40 minutes per day (p. 778).

\subsection{Tobacco Use}

Patel et al. (2015) stated that tobacco use is one of the top leading risk factors for cardiovascular death in the United States for both men and women (p. 245). However, it is not just smoking tobacco that impacts CVD; Gupta, Gupta, and Khedar (2013) found that smokeless tobacco such as chew or snuff had similar devastating effects on the cardiovascular system (p. 370). Gupta et al. discussed how the use of smokeless tobacco increased the risk for other diseases leading to cardiovascular events, such as metabolic syndrome, insulin resistance, and hypercholesterolemia. The risk for development may increase by $2.5 \%$. Other negative effects of smoking on the cardiovascular system include dysfunction of platelet activation, thrombogenesis, and endothelial dysfunction (Gupta et al., 2013, pp. 374-375).

\subsection{Dietary Factors}

Diets consisting of high fat and sodium content increase the risk of experiencing a cardiovascular event. Multiple studies have shown that diets consisting of fruits, vegetables, nuts, unrefined grains, legumes, fish, and olive oil have beneficial associations with decreasing the risk for CVD (Robson, 2014). Dietary consumption of foods rich in flavonoids such as grapes, wine, teas, soy and dark chocolate have shown to enhance endothelial function while decreasing blood pressure. In another study, El--Rei et al. (2013) found that after consuming dark chocolate for seven days, participants experienced a decrease in systolic blood pressure from $140 \mathrm{mmHg}$ to 131 $\mathrm{mmHg}$ and a diastolic reading from $85 \mathrm{mmHg}$ to $82 \mathrm{mmHg}$. El--Rei et al. concluded that hypertensive patients showed a "significant improvement of $2.3 \%$ in endothelial function" without drug therapy, after incorporating dark chocolate into their diet for a total of 15 days (p. 3). Dark chocolate, however, caused little improvement in "vascular stiffness" (p. 4). Through the meta-analysis study by El-Rei, it was discovered that consuming dark chocolate, and only dark chocolate, reduced CVD by $31 \%$ (El--Rei et al., 2013, p. 5).

\subsection{Age}

The risk for developing CVD inevitably increases as one ages, which is due to several factors. Physiologically, one experiences changes in the cardiovascular system, which could partially be attributed to issues such as atherosclerosis or stiffening of the blood vessels or myocardial tissue. Additionally, it is not uncommon for people to experience increases in cholesterol levels and blood pressure as they age (Ignatavicius \& Workman, 2013). Such changes may be reduced to a certain degree with control of modifiable risk factors, but a functional decline is not entirely avoidable.

Modig, Andersson, Drefahl, and Ahlbom (2013) conducted a study to determine the degree to which the risk for CVD and myocardial infarction (MI) event increases with each year that one advance in age. This study was conducted retrospectively through a myriad of hospital databases to examine the number of CVD and MI diagnoses correspondent to age. The increase in relative risk in age for CVD and mortality was determined through a regression model comparative between each year of age. Based on the study, "the relative risk for a 
CVD event per one year increase in age was about $9 \%$ for every successive year (for both men and women) between 85 to 94 years," (Modig et al., 2013, p. 3) which is a significant increase in risk as one advances in age. The impact of age on CVD risk is irrefutable, but unfortunately, this factor serves as an unmodifiable risk of development. Such a factor is especially important for females to understand, as their risk increases significantly with progressing age and accompanying postmenopausal changes.

\subsection{Gender}

Mosca, Barrett-Connor, and Wenger (2015) found that risk factors between genders remain the same, but the prevalence of risk factors differs per gender. Gender serves as a significant, unmodifiable risk factor for CVD, with risk increasing for females after menopause. Before menopause, women are at a lower risk for developing CVD than their male counterparts, according to data obtained from the National Health and Nutrition Examination Surveys (Mosca et al., 2015, p. 2148). Mosca et al. thoroughly analyzed surveys from a study comparing the risk of CVD relative to gender risk associated with age. The changes females may experience during menopause increases the prevalence of certain risk factors. Because of hormonal changes and deficiencies in estrogen, women may experience an increase in BMI, hypercholesterolemia, hypertension, diabetes mellitus, and other metabolic changes (Maas \& Appelman, 2011, p. 599). Understanding physiologic changes that occur during menopause is important for women, as the risk for CVD increases significantly during this time.

\subsection{Ethnicity}

Ethnicity serves as a significant risk factor for CVD because of many intricate factors, such as genetic disposition towards certain risk factors, various health disparities, and environmental factors precipitating increased risk of modifiable factors. People of a certain race or ethnicity may be genetically predisposed toward certain risk factors, such as hypertension, hypercholesterolemia, or metabolic disturbances leading to diabetes mellitus (Maas \& Appelman, 2011, p. 599).

Mozaffarian et al. (2015) performed a statistical analysis of the prevalence of CVD in the U.S. and worldwide. The analysis provided information about the influence that risk factors such as ethnicity/race and gender exerted on the development of CVD. Many ethnic/racial minority populations are at a higher risk for CVD consequently, giving blacks, American Indians/Alaskan Natives, Hispanics, and Mexican-Americans a higher risk in certain categories. For example, "the prevalence of CVD among women $>20$ years of age is $47 \%$ among blacks, $34 \%$ among whites, and 31\% among Mexican Americans" (Mozaffarian et al., 2015, p. 1) with the prevalence of hypertension being highest in the black population. The high prevalence of certain risk factors within a race/ethnicity may lend toward its increased incidence within the population.

Additionally, certain environmental factors may precipitate an increased risk of modifiable behaviors. Certain racial or ethnic groups may be more inclined toward performing certain behaviors, such as smoking or obesity. For example, African-Americans (non-Hispanic),

Mexican-Americans, American-Indians, and Alaska Natives have a higher incidence of diabetes than Caucasian, and non-Hispanic African-American and Mexican-American women are reported to have a higher rate of obesity (Mozaffarian et al., 2015). The environment may contribute to a reduction in the performance of behaviors that could reduce modifiable risk factors for CVD. Such could be due in part to lower income and educational levels of certain racial or ethnic minorities.

People of lower socioeconomic status may not have access to health care or the resources necessary to perform healthy behaviors, leading to an increased risk for CVD development. Mozaffarian et al. (2015) observed, "In 2008, cardiovascular deaths represented 30 percent of all global deaths, with 80 percent of those deaths taking place in low- and middle-income countries" (p. 1). This reflects the importance of education and resources toward reducing health disparities that lead to an increase in the prevalence of CVD. Certain aspects of ethnicity serve as a non-modifiable risk factor for CVD, as the race may cause genetic differences that directly increase one's risk. Health disparities and environmental factors increasing modifiable risks, however, may be altered to reduce risk within a certain racial population.

\subsection{Family History}

Family history is an important risk factor for CVD because a positive family history of a cardiovascular event increases an individual's risk "5-fold" (Kashani, Eliasson, Vernalis, Bailey, \& Terhaar, 2015, p. 292). Kashani et al. defined positive family history as a first-degree relative (i.e. biological parents, full siblings, or children) experiencing a CVD event before the age of 55 for males, and 65 years of age for females. The Framingham Risk Score is used to predict the level of risk for the development of a cardiovascular event; however, this tool does not incorporate family history as defining criteria when computing a score. This deficiency of information 
can have serious consequences by understating an individual's level of risk. Kashani et al. advocated for practitioners to not only consider an individual's Framingham score but to also take into account the individual's family history.

\subsection{Summary of Literature Review}

In summary, non-modifiable and modifiable risk factors for CVD were discussed in depth in the literature reviewed. These peer-reviewed articles contributed to the understanding of CVD risk factors and their impact on a given population and individual. Understanding the relevance of each factor was important before conducting a research study investigating college students' perception of risk factors. Considering the devastating outcomes associated with CVD, the literature indicated the importance of reducing modifiable risk factors associated with CVD. This makes it imperative for healthcare professionals to be proactive partners in promoting lifestyle changes. However, change cannot begin until individuals are empowered with the knowledge surrounding CVD, thus eliminating their knowledge deficit while enhancing positive lifestyle and behavioral changes.

\section{Definitions}

CVD can be defined as any disease about the heart and blood vessels, which encapsulates a myriad of conditions. CVD is essentially an umbrella term for a diverse range of conditions. Arteriosclerosis, coronary heart disease, congenital heart disease, heart failure, valvular diseases, inflammatory heart disease, arrhythmias, peripheral artery disease, and deep vein thrombosis are all considered types of cardiovascular disease. For this research study, however, CVD will refer to any condition affecting the function of the heart, without delving into specifics about the various types. Risk factors are attributes or characteristics that increase the likelihood of developing a disease or condition. Examples of risk factors about CVD could include, but are not limited to, the following: obesity, hypertension, physical inactivity, and hypercholesterolemia.

Modifiable risk factors are risk factors that are alterable and possess the ability to either increase or decrease one's likelihood of developing a disease or condition. Examples of modifiable risk factors for CVD could include issues such as weight, activity, diet, and smoking cessation. Instigating behavioral changes directed towards eradicating or decreasing modifiable risk factors may decrease one's likelihood of developing a condition or exacerbating a preexisting condition. Non-modifiable risk factors are unalterable risk factors that may increase one's likelihood of developing a disease or condition. Characteristics such as age, race, or gender may increase one's risk for development, but these factors cannot be eradicated by behavioral changes.

\section{Methodology}

A quantitative study was conducted; this approach allowed the researchers to obtain information from a vast student population. A broad range of 20 Likert-type statements about CVD risk factors was presented with limited pre-selected responses. To accommodate for voluntary participation and convenience, Likert-type statements were most appropriate to use, as these statements were arranged in an organized, systematic form that was simple to complete. A sample size of 118 college students with various demographics was obtained. Participation in this research study was random, strictly voluntary, and anonymous. Participants varied in gender, ethnicity, and age. All participants were a minimum age of 18 years to ensure that participants were of legal age to ethically participate in the study. The geographic location of the survey was a college in the Midwestern region of the United States.

The survey instrument was created after a detailed review of the literature. The survey tool in this research study included twenty statements that related to the research question and five demographic questions. Participants had to answer each statement based on the Likert-type scale used. It included responses of strongly disagree (SD), disagree (D), agree (A), and strongly agree (SA). These responses had numeric values assigned to them with SD being valued as one and SA having a value of four.

The College Institutional Review Board (IRB) approval was obtained before conducting research. Participants were asked to sign an informed consent prior to participation and were provided a copy for their records. Data collected from the surveys was kept anonymous by excluding participants' names and identifying information from the surveys.

\section{Results}

One primary research question guided the study: What is college students' knowledge of risk factors related to cardiovascular disease? The results of the analyses appear in the following sections. The mean and standard deviation were calculated. The standard deviation took into account the values of each score and provided a comparison between scores. 


\subsection{Demographic Characteristics}

Table 1 contains the descriptive statistics for the participants' demographics. The most common age ranges were $18-20(71 \%)$ and 21-23 (25\%). More females (58\%) than males (42\%) were surveyed. With a total sample size of 118 participants, the freshman students accounted for over a third of those surveyed (38\%) and junior students followed with the next highest participation (27\%). The majority of the participants were White/Caucasian (79\%). Many of the participants denied a family history of heart disease (47\%), about a third expressed knowledge of family history of heart disease (30\%), and the remaining did not know if there was a family history of heart disease or not $(23 \%)$.

Table 1. Descriptive Statistics for Participant Demographics and Background

\begin{tabular}{|c|c|c|}
\hline Variable & $f$ & $\%$ \\
\hline \multicolumn{3}{|l|}{ Gender } \\
\hline Male & 49 & 42 \\
\hline Female & 69 & 58 \\
\hline \multicolumn{3}{|l|}{ Age Range } \\
\hline $18-20$ & 84 & 71 \\
\hline $21-23$ & 29 & 25 \\
\hline $24-26$ & 5 & 4 \\
\hline \multicolumn{3}{|l|}{ Class } \\
\hline Freshman & 45 & 38 \\
\hline Sophomore & 21 & 18 \\
\hline Junior & 32 & 27 \\
\hline Senior & 19 & 16 \\
\hline Graduate Student & 1 & $<1$ \\
\hline \multicolumn{3}{|l|}{ Race } \\
\hline White/Caucasian & 93 & 79 \\
\hline African American & 10 & 8 \\
\hline Hispanic/Latino & 5 & 4 \\
\hline Asian & 4 & 3 \\
\hline Other & 6 & 5 \\
\hline \multicolumn{3}{|c|}{ Family History of Cardiovascular Heart Disease } \\
\hline Yes & 35 & 30 \\
\hline No & 56 & 47 \\
\hline Unknown & 27 & 23 \\
\hline
\end{tabular}

Note. $(\mathrm{N}=118)$.

\subsection{Item Set Description}

Each of the 20 statements in the study was designed to assess students' knowledge about risk factors related to CVD. Participants selected one of the four selections to indicate the statement that most accurately addressed their personal knowledge. In Table 2, the mean levels of frequency of each of the twenty survey statements are ranked by the level of frequency. Each of the items had means between 1.67 and 3.52. The two statements the participants unanimously agreed with were the smoking tobacco statement, "Smoking tobacco increases my risk 
for developing CVD" (M=3.52, $\mathrm{SD}=0.66)$ and the active lifestyle statement, "Maintaining an active lifestyle will reduce my risk for CVD" $(\mathrm{M}=3.52, \mathrm{SD}=0.52)$. Participants additionally tended to agree with the statements, "Hypertension (high blood pressure) is a significant source of CVD" $(\mathrm{M}=3.36, \mathrm{SD}=0.59)$ and "Increasing fresh fruits and vegetables in my diet decreases my risk for CVD" $(\mathrm{M}=3.36, \mathrm{SD}=0.56)$. Participants also agreed with the statement, "Elevated cholesterol levels increase my risk for developing CVD" $(\mathrm{M}=3.31, \mathrm{SD}=0.57)$.

The statement with the lowest mean was, "Weight has little influence in the development of CVD" $(\mathrm{M}=1.67$, $\mathrm{SD}=0.85$ ). Even though the mean is low for this statement, it demonstrates participants' knowledge that obesity is a risk factor for CVD because they disagreed with this statement. Another statement with a low mean was, "Chewing tobacco decreases my risk of developing CVD" $(\mathrm{M}=1.75, \mathrm{SD}=0.94)$. This also indicates the understanding that chewing tobacco is a risk factor of CVD as there was disagreement with this statement as well. Participants also disagreed with the statement, "Women develop CVD more rapidly after the onset of menopause" $(\mathrm{M}=2.54, \mathrm{SD}=0.61)$. However, this indicates a lack of knowledge, as women are more prone to CVD after the onset of menopause. Another statement that demonstrated a lack of knowledge was, "My ethnicity is a risk factor for CVD" $(\mathrm{M}=2.58, \mathrm{SD}=0.76)$. College students were inclined to disagree with this statement signifying they are not aware that ethnic minorities are at a higher risk for development of CVD. After determining the means for all twenty research statements, there were five areas college students exhibited the greatest knowledge of risk factors related to CVD. The highest recognized risk factors were smoking tobacco, inactive lifestyle, hypertension, decreased consumption of fresh fruits and vegetables, and elevated cholesterol.

Table 2. Cardiovascular Risk Factors

\begin{tabular}{|c|c|c|}
\hline Variable & $M$ & $S D$ \\
\hline Smoking tobacco increases my risk for developing CVD. & 3.52 & .66 \\
\hline Maintaining an active lifestyle will reduce my risk for CVD. & 3.52 & .52 \\
\hline Hypertension (high blood pressure) is a significant source of CVD. & 3.36 & .59 \\
\hline Increasing fresh fruits and vegetables in my diet decreases my risk for CVD. & 3.36 & .56 \\
\hline Elevated cholesterol levels increase my risk for developing CVD. & 3.31 & .57 \\
\hline The amount of dietary sodium (salt) I consume may increase my risk of CVD. & 3.25 & .61 \\
\hline As my age increases, my risk for developing CVD increases. & 3.22 & .49 \\
\hline Consuming a diet high in saturated fat increases my risk for CVD. & 3.18 & .77 \\
\hline My risk of CVD increases with raised glucose (sugar) levels in my blood. & 3.02 & .55 \\
\hline Sleeping at least 8 hours per night decreases my risk of developing CVD. & 3.00 & .63 \\
\hline Drinking at least 8 cups of water a day will decrease my risk of developing CVD. & 2.98 & .55 \\
\hline $\begin{array}{l}\text { My family's history of Cardiovascular Disease (CVD) increases my risk for } \\
\text { developing CVD. }\end{array}$ & 2.98 & .55 \\
\hline Consuming dark chocolate can help lower my risk for CVD. & 2.70 & .57 \\
\hline Lower economic status increases the risk for developing CVD. & 2.63 & .77 \\
\hline My ethnicity is a risk factor for CVD. & 2.58 & .76 \\
\hline Women develop CVD more rapidly after the onset of menopause. & 2.54 & .61 \\
\hline My gender does not increase my risk for developing CVD. & 2.19 & .79 \\
\hline Cold weather contributes to my risk of developing CVD. & 2.03 & .52 \\
\hline Chewing tobacco decreases my risk of developing CVD. & 1.75 & .94 \\
\hline Weight has little influence in the development of CVD. & 1.67 & .85 \\
\hline
\end{tabular}

Note. ( $\mathrm{N}=118$ ). Items were rated on a 4-point Likert-type scale ranging from 1 (Strongly disagree) to 4 (Strongly Agree), so higher mean indicates higher levels of agreement. 


\section{Discussion}

The results of this quantitative study were compared to the literature review. The survey data analysis indicated that most college students are aware of the main CVD risk factors. The majority of the students displayed knowledge of risk factors such as smoking tobacco, inactive lifestyle, hypertension, low intake of fruits and vegetables, elevated cholesterol levels, high dietary sodium obesity, and other pertinent risk factors. A knowledge deficit existed toward gender and ethnicity, which are non-modifiable risk factors, albeit important considerations.

Forty-five respondents (38\%) agreed, and 68 respondents (58\%) strongly agreed that smoking tobacco increases the risk for the development of CVD. Additionally, $80 \%$ of the students surveyed displayed knowledge that chewing tobacco can also increase risk. Patel et al. (2015) reported that tobacco use is one of the top leading risk factors for cardiovascular death in the United States for both men and women (p. 245). This is a very modifiable risk factor, and a major contributor to CVD, so knowledge about it is important. College students exhibit knowledge about the impact of smoking on cardiovascular health, as evidenced by the survey responses. Clearly, students are not ignorant of this risk factor, though it is undetermined how many individuals will still choose to smoke despite the risks.

Students also appear knowledgeable about the impact that dietary choices can exert on cardiovascular health. Sixty-six participants agreed (56\%) and 47 strongly agreed (40\%) that increasing fruits and vegetables in the diet decreases risk for CVD. Additionally, $86 \%$ of students were in agreement that saturated fat can increase risk, and $92 \%$ expressed understanding that increased dietary sodium can also contribute. Seventy-one participants (60\%) agreed, and 40 participants (34\%) strongly agreed that elevated cholesterol levels contribute to an increased risk. Despite the positive responses pertaining to diet, it is unclear just how substantiated this knowledge is for students. Students may be aware that a diet high in fruits and vegetables is beneficial, but perhaps the meaning of this statement varies per individual. For example, one student may consider one piece of fruit and a vegetable to constitute a high daily intake, while another student may believe that high intake would entail fruits and vegetables composing the majority of his or her diet. Furthermore, students may be knowledgeable that high saturated fat, sodium, and cholesterol can negatively impact health, but their knowledge may not extend beyond that fact. It is possible that many students are unaware of the daily recommended intake values for sodium or foods that contain a high amount of saturated fats, or the recommended reference range for blood cholesterol levels. This study was limited in investigating the depth of knowledge and was only able to identify areas of general knowledge strength and weaknesses.

Another area of knowledge strength that was identified pertained to the importance of an active lifestyle for CVD prevention. Fifty-five participants (47\%) agreed and 62 participants (53\%) strongly agreed that maintaining an active lifestyle reduces the risk for CVD. A study conducted by Kozey-Keadle et al. (2014) supports this concept by exploring the impact of exercise on the cardiovascular system. The study concluded that exercise is beneficial for the reduction of BMI, blood pressure, and triglyceride levels in participants (p. 774). Exercise significantly reduces one's risk for the development of CVD by decreasing other areas of risk, like the factors above. Students may or may not be knowledgeable about how exercise reduces risk, or how much exercise is necessary to achieve risk reduction goals, but regardless, students appear knowledgeable about the general importance of an active lifestyle for cardiovascular health.

Hypertension is another area of strength of which students appeared knowledgeable. Notably, $97 \%$ of students were in agreement that hypertension is a significant source of CVD. While it is apparent that students understand that high blood pressure is a major contributor, knowledge variances could exist, as students may have differing definitions of what constitutes elevated blood pressure. Hypertension is defined as an average systolic blood pressure greater than $140 \mathrm{~mm} \mathrm{Hg}$, and an average diastolic blood pressure greater than $90 \mathrm{~mm} \mathrm{Hg}$ (Patel et al., 2015 , p. 246). These are the recognized values constituting hypertension, but it remains unclear whether or not students' definitions align with this standard. Students may not possess knowledge about what constitutes high blood pressure so definitions may vary between individuals. Regardless, students still appear to possess a general knowledge about the negative impact that hypertension can exert on cardiovascular health. Only three students surveyed $(<3 \%)$ were in disagreement about this risk factor.

Patel et al. (2015) found that half of national cardiovascular deaths can be connected to the combination of the following five risk factors: elevated cholesterol levels, diabetes, hypertension, obesity, and smoking (pp. 248-249). College students demonstrated knowledge about the above risk factors, according to this research study. Certain knowledge deficits were identified, however, specifically about two of the non-modifiable risk factors. Ethnicity and gender were the risk factors receiving the lowest positive responses in the current study. These risk factors are 
non-modifiable, so no health behaviors on the part of the student can assist in lowering this risk; however, awareness is still important.

As it pertains to gender, $50 \%$ of the students surveyed were in agreement that risk for CVD increases for women after the onset of menopause. Fifty-eight respondents $(49 \%)$ disagreed and one respondent $(<1 \%)$ strongly disagreed with this statement, however, signifying a deficit of knowledge in this area. Of the fifty-eight respondents who disagreed, $55 \%$ were female. Since $46 \%$ of the total female population answered negatively to this statement, education about CVD risk after menopause would be beneficial. In a retrospective study, Mosca et al. (2015) found, the risk for females increases significantly after menopause due to the physiological changes that occur for women at this developmental stage (p. 2148). Twenty-two percent of the respondents agreed, and 7\% strongly agreed that gender does not increase the risk for CVD while $71 \%$ of students disagreed with this statement. Although the majority of the students did express understanding, "My gender does not increase my risk for developing CVD" $(\mathrm{M}=2.19, \mathrm{SD}=0.79)$ and "Women develop CVD more rapidly after the onset of menopause" $(\mathrm{M}=2.54, \mathrm{SD}=0.61)$ were the risk factors with the lowest means in the current study. It is important for students to understand the risk factors associated with certain non-modifiable physical aspects about themselves, such as age or gender so that those with increased risk can exercise further caution in the areas where modifications can occur.

Ethnicity was another non-modifiable risk factor of which students did not appear to be knowledgeable. Sixty percent of students agreed or strongly agreed that ethnicity is a risk factor for CVD, while $32 \%$ of students disagreed and $8 \%$ strongly disagreed with this statement. Of the ten African-American students who participated in the survey, only one disagreed $(10 \%)$ with this statement; the majority agreed $(80 \%)$ or strongly agreed $(10 \%)$ that ethnicity is a risk factor. Mozaffarian et al. (2015) stated, "the prevalence of CVD among women $>20$ years is $47 \%$ among blacks, 34\% among whites, and 31\% among Mexican Americans" (p. 1). Many ethnic populations experience a higher risk for CVD because of the increased incidence of certain factors, such as hypertension, among these populations. The majority of students surveyed were White/Caucasian (79\%), with $8 \%$ of the respondents describing themselves as African-American, 4\% as Latino/Hispanic, 3\% as Asian, and 5\% as other. It is important for each person to understand the significance that his or her ethnicity may play in the development of CVD so that health behaviors can be addressed to reduce modifiable risks.

In summary, the results indicated that college students have a beneficial knowledge of risk factors related to CVD. They managed to agree with a majority of statements that indicated risk factors for CVD, such as smoking tobacco and inactive lifestyle, and disagree with incorrect statements, for example chewing tobacco decreases risk and weight have little influence. However, there were a few statements that demonstrated a lack of knowledge of risk factors related to CVD, for instance, women after the onset of menopause and ethnic minorities.

\section{Implications and Recommendations}

The promotion of cardiovascular health is an important element of nursing, as a large proportion of deaths worldwide can be attributed to this condition. Reducing the number of incidences, and consequently reducing the number of related deaths, can be accomplished by practicing prevention. Patient preventive action, such as lifestyle modifications, can be instigated with the assist of nurse preventive action, such as education. Educating patients about risk factors will empower them with the knowledge required to help reduce modifiable risks. This research study identified areas of knowledge deficit, which could potentially be utilized to help direct the focus of educational goals.

The strong areas of knowledge identified, about risk factors such as hypertension and diet, could also benefit from further exploration. Although students displayed knowledge of these risk factors, the depth of their knowledge is not evident. Even though students appear knowledgeable about areas such as cholesterol in cardiovascular health, further education could be beneficial to improve this knowledge base. Teaching topics could include, but are not limited to types of foods high in cholesterol, cholesterol-lowering pharmacological agents, appropriate cholesterol blood levels, the difference between LDL and HDL cholesterol, and non-pharmacological methods for the reduction of cholesterol. Such topics of education delve into the details of each risk factor, further equipping students with the knowledge necessary for the implementation of beneficial health changes. Education is one of many possible steps in prevention of cardiovascular disease. Early prevention and early detection are imperative for the potentiation of health, and the results of the current study can help achieve this by identifying strong and weak knowledge areas.

Further research should be conducted to evaluate the knowledge of CVD risk factors in the general population, as well as other colleges. The community and the type of college may negatively or positively impact the amount of knowledge that an individual possesses about CVD. The current study was restricted in that the population of this college is rather homologous, and the results of the study pertain specifically to this college campus. 
Conducting similar research in different environments could yield differing results and identify knowledge deficits specific to the chosen populations. Results could be compared between communities or between colleges to identify commonalities and areas that require additional education.

\section{Acknowledgement}

The authors wish to acknowledge Dr. Deborah Gillum, the Dean of Nursing, Bethel College, Indiana, for offering valuable comments during the early stages of the study. We also like to thank Bethel College administrators for giving us the opportunity to conduct this study.

\section{References}

El-Rei, J. D., Cunha, A. R., Burla, A., Burla, M., Oigman, W., Neves, M. F., \& Virdis, A. (2013). Characterisation of hypertensive patients with improved endothelial function after_dark chocolate consumption. International Journal of Hypertension, (2013), 1-6. https://doi.org/10.1155/2013/985087

Gupta, R., Gupta, N., \& Khedar, R. S. (2013). Smokeless tobacco and cardiovascular disease in low and middle-income countries. Indian Heart Journal, 65(4), 369-377. https://doi.org/10.1016.j.ihj.2013.06.005

Ignatavicius, D., \& Workman, M. (2013). Medical-surgical nursing: Patient-centered collaborative care (7th ed.), pp. 772-1413. St. Louis: Elsevier Saunders.

Kashani, M., Eliasson, A., Vernalis, M., Bailey, K., \& Terhaar, M. (2015). A systematic approach incorporating family history improves identification of cardiovascular disease risk. Journal of Cardiovascular Nursing, 30(4), 292-297. https://doi.org/10.1097/JCN.0000000000000163

Kozey-Keadle, S., Lynden, K., Staundenmayer, J., Hickey, A., Viskochil, R., Braun, B., \& Freedson, P. S. (2014). The independent and combined effects of exercise training and reducing sedentary behavior on cardiometabolic risk factors. Applied Physiology, Nutrition \& Metabolism, 39(7), 1-12. https://doi.org/10.1139/apnm-2013-0379

Maas, A. H., \& Appelman, Y. E. (2010). Gender differences in coronary heart disease. Netherlands Heart Journal, 18(12), 598-602. https://doi.org/10.1007/s12471-010-0841-y

Melnyk, J., Panza, G., Zaleski, A., \& Taylor, B. (2015). Awareness and knowledge of cardiovascular risk through blood pressure and cholesterol testing in college freshmen. American Journal of Health Education, 46(3), 138-143. https://doi.org/10.1080/19325037.2015.1023474

Modig, K., Andersson, T., Drefahl, S., \& Ahlbom, A. (2013). Age-specific trends in morbidity, mortality, and case-fatality from cardiovascular disease, myocardial infarction, and stroke in advanced age: Evaluation in the Swedish population. PLOS ONE, 8(5), 1-12. https://doi.org/10.1371/journal.pone.0064928

Mosca, L., Barrett-Connor, E., \& Wenger, N. (2015). Sex/gender differences in cardiovascular disease prevention. American Heart Association Journals, 2145-2152. https://doi.org/10.1161/CIRCULATIONAHA.110.968792

Mozaffarian, D., Benjamin, E. J., Go, A. S., Arnett, D. K., Blaha, M. J., Cushman, M., \& Turner, M. B. (2015). Heart disease and stroke statistics - 2015 update: A report from the American Heart Association. Circulation, 1-3. https://doi.org/10.1161/CIR.0000000000000152

Patel, S. A., Winkel, M., Ali, M. K., Narayan, K. V., \& Mehta, N. K. (2015). Cardiovascular mortality associated with 5 leading risk factors: National and state preventable fractions estimated from survey data. Annals of Internal Medicine, 163(4), 245-253. https://doi.org/10.7326/M14-1753

Robson, D. (2014). Positive effects of the Mediterranean diet in the prevention and management of cardiovascular disease: A literature review. Journal of the Australian Traditional-Medicine Society, 20(3), $1-5$.

\section{Copyrights}

Copyright for this article is retained by the author(s), with first publication rights granted to the journal.

This is an open-access article distributed under the terms and conditions of the Creative Commons Attribution license (http://creativecommons.org/licenses/by/4.0/). 